\title{
Potato virus $Y$ (PVY) Isolates from Physalis peruviana are Unable to Systemically Infect Potato or Pepper and Form a Distinct New Lineage Within the PVYC Strain Group
}

\author{
Kelsie J. Green, Mohamad Chikh-Ali, Randall T. Hamasaki, Michael J. Melzer, and Alexander V. Karasev ${ }^{\dagger}$
}

First, second, and fifth authors: Department of PSES, University of Idaho, Moscow; third and fourth authors: University of Hawaii at Manoa, Honolulu; and fifth author: and Bioinformatics and Computational Biology Program, University of Idaho, Moscow.

Accepted for publication 24 June 2017.

\begin{abstract}
Poha, or cape gooseberry (Physalis peruviana L.), is a plant species cultivated in Hawaii for fresh fruit production. In 2015, an outbreak of virus symptoms occurred on poha farms in the South Kohala District of the island of Hawaii. The plants displayed mosaic, stunting, and leaf deformation, and produced poor fruit. Initial testing found the problem associated with Potato virus $Y$ (PVY) infection. Six individual PVY isolates, named Poha1 to Poha6, were collected from field-grown poha plants and subjected to biological and molecular characterization. All six isolates induced mosaic and vein clearing in tobacco, and three of them exhibited O-serotype while the other three reacted only with polyclonal antibodies and had no identifiable serotype.

Until now, PVY isolates have been broadly divided into pepper or potato adapted; however, these six PVY isolates from poha were unable to establish systemic infection in pepper and in four tested potato cultivars. Wholegenome sequences for the six isolates were determined, and no evidence of recombination was found in any of them. Phylogenetic analysis placed poha PVY isolates in a distinct, monophyletic "Poha" clade within the PVYC lineage, suggesting that they represented a novel, biologically and evolutionarily unique group. The genetic diversity within this poha PVYC clade was unusually high, suggesting a long association of $\mathrm{PVY}^{\mathrm{C}}$ with this solanaceous host or a prolonged geographical separation of $\mathrm{PVYC}^{\mathrm{C}}$ in poha in Hawaii.
\end{abstract}

Physalis peruviana L., named poha or poha berry in Hawaii and Peruvian groundcherry and cape gooseberry in Europe, South Africa, and Australia, is a solanaceous plant cultivated in Hawaii for its sweet fruit, which can be eaten fresh or used in food processing to produce ice cream, salsa, or jam. The plants can be propagated from seed or using cuttings, and are typically grown as an annual crop in greenhouses, screenhouses, or open fields at higher elevations in the South Kohala and Puna Districts of the island of Hawaii. The high market value of poha fruit, which can exceed $\$ 18 / \mathrm{kg}$, is primarily due to its scarcity in local markets despite the efforts of many growers to cultivate this crop. Fruit production in Hawaii has long been hindered by a viral disease which has contributed to the abandonment of several farms in recent years, particularly in South Kohala. Affected plants are stunted and exhibit deformed and discolored husks, leaf mosaic, and various leaf deformations. This disease was found to be associated with the presence of Potato virus $Y$ (PVY) based on serological and molecular evidence but the strain assignment for the virus causing this disease in poha crops was not conducted (Hamasaki et al. 2015).

PVY is the type member of the genus Potyvirus, family Potyviridae, having a single-stranded, positive-sense RNA genome of $9.7 \mathrm{~kb}$, excluding the poly(A) tail, encapsidated in a flexuous filamentous particle, and is transmitted in nature nonpersistently by multiple species of aphids (Adams et al. 2012). PVY exists as a complex of strains that are defined based on their host range in several solanaceous plants and also on their molecular characteristics (Karasev and Gray 2013; Kerlan 2006; Quenouille et al. 2013; Singh et al. 2008). Phylogenetically, all nonrecombinant PVY isolates fall into four main evolutionary lineages, named PVYO, PVYN, PVYC, and Chile3,

†Corresponding author: A. V. Karasev; E-mail: akarasev@uidaho.edu

*The $\boldsymbol{e}$-Xtra logo stands for "electronic extra" and indicates that one supplementary figure and one supplementary table are published online.

(C) 2017 The American Phytopathological Society which correspond with their host range characteristics (Quenouille et al. 2013; Singh et al. 2008). The PVYO and PVYN lineages comprise potato isolates which are not well adapted to pepper, whereas the PVYC lineage comprises predominantly pepper isolates which are not well adapted to potato. The Chile 3 lineage comprises a single pepper isolate unable to infect potato (Moury 2010). The PVYC lineage is very diverse and, based on phylogenetic analyses, has been subdivided into clades $\mathrm{C} 1$ and $\mathrm{C} 2$, with the $\mathrm{C} 1$ clade representing nonpotato isolates (Blanco-Urgoiti et al. 1998; Moury 2010). However, the C1 clade was later demonstrated to include at least one potato-infecting isolate, PRI-509 (Dullemans et al. 2011). Recently, we described a pepper PVYC isolate on the island of Oahu, Hawaii, isolated from a commercially cultivated field tomato plant which was unable to infect potato and phylogenetically was placed in the $\mathrm{C} 1$ clade (Chikh-Ali et al. 2016).

Until now, all characterized PVY isolates could be classified as either pepper or potato adapted, with other solanaceous hosts such as tobacco (Nicotiana tabacum L.), N. benthamiana, and tomato (Solanum lycopersicum L.), serving as intermediate permissive hosts (Chikh-Ali et al. 2016; Dullemans et al. 2011; Kerlan 2006; Quenouille et al. 2013; Singh et al. 2008). Here, we characterized six field PVY isolates from a novel solanaceous host, poha or cape gooseberry, which were found unable to systemically infect either potato or pepper. The whole-genome sequences of these six PVY isolates formed a distinct phylogenetic "Poha" clade within the PVYC lineage. These PVYC sequences from the Poha clade exhibited an unexpectedly high level of divergence, suggesting prolonged adaptation of PVY to this host or a long geographic isolation of PVYC in Hawaii.

\section{MATERIALS AND METHODS}

Virus isolates: origin and maintenance. In June 2015, 21 poha leaf samples displaying symptoms of virus infection were collected from two locations, $1.6 \mathrm{~km}$ apart, within the South Kohala District of the island of Hawaii. These samples were evaluated for 
the presence of PVY using an immunostrip assay (Agdia). Following this initial mini-survey, six poha isolates, designated Poha1 through Poha6, were collected in August 2015 from infected poha plants growing on a commercial farm in Waimea, on the island of Hawaii. This commercial farm was one of the two locations sampled 2 months earlier and determined to have PVY-positive poha plants. The infected plants displayed symptoms typical of PVY infection in $P$. peruviana, which included mosaic, crinkling, and leaf deformation (Fig. 1). These original isolates were inoculated into tobacco (N. tabacum L., 'Burley') and confirmed to be systemically infected with PVY. These Poha PVY isolates were maintained in tobacco through periodic reinoculations to young healthy plants in an insectfree growth room as part of the PVY isolate collection maintained at the University of Idaho (UI). This collection was periodically subjected to serological (Nikolaeva et al. 2012) and reverse-transcription polymerase chain reaction (RT-PCR) (Chikh-Ali et al. 2013; Lorenzen et al. 2006) typing as part of the maintenance protocol to avoid contamination or strain mixtures and ensure stable characteristics of the reference isolates. Three PVY isolates from the PVYC strain group were used as biological controls: H14 (GenBank accession KX580384) (Chikh-Ali et al. 2016), AL100001 (KY847935) (Green et al. 2017), and NY110001 (KY848014) (Green et al. 2017). The two reference PVY isolates Oz (strain PVYO; EF026074), and N605 (strain PVYN; X97895) were used for comparisons.

Biological characterization. The following indicator plants were used: Capsicum annuum 'Early California Wonder' (pepper), N. tabacum Burley (tobacco), and N. benthamiana. All plants were mechanically inoculated with sap from PVY-infected tobacco at the four- to six-leaf stage, as described previously (Chikh-Ali et al. 2016; Hu et al. 2009). Two potato cultivars with known genetic

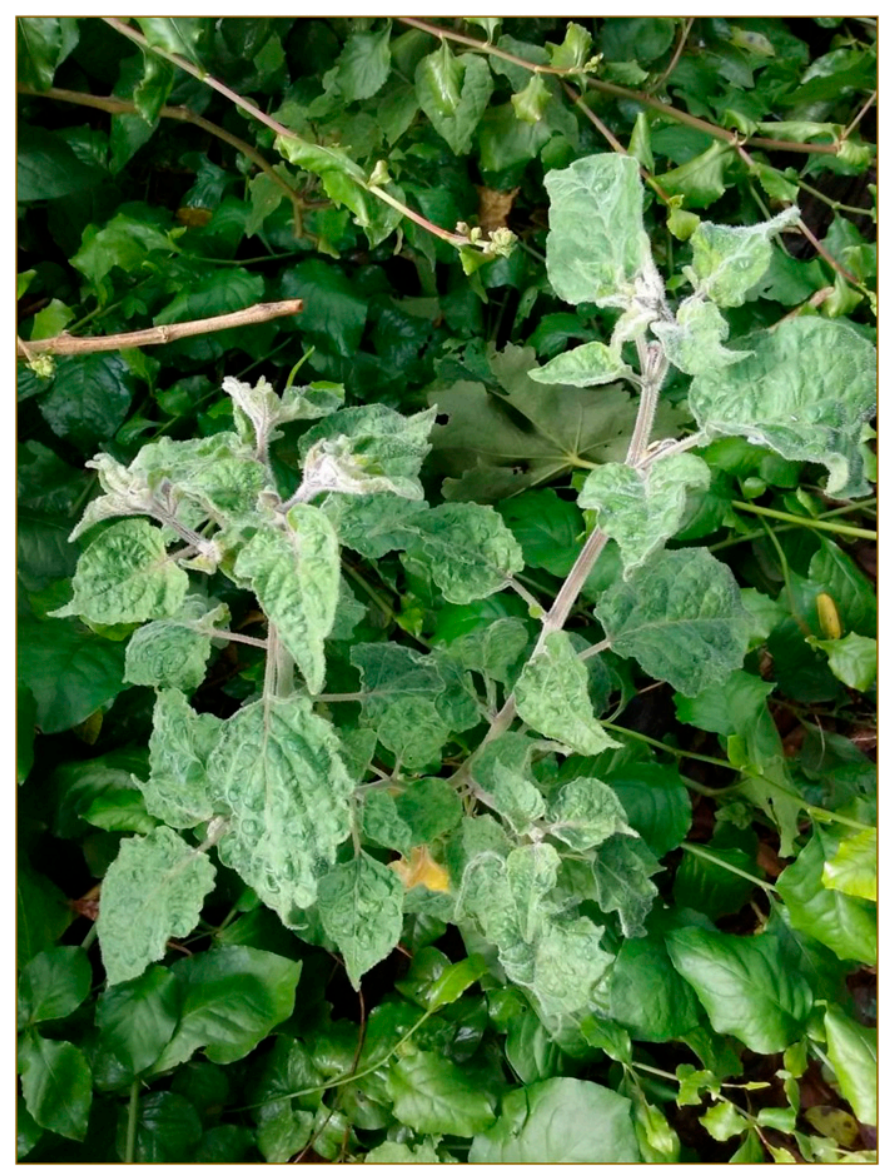

Fig. 1. Symptoms of mosaic, crinkling, and leaf deformation induced by Potato virus $Y$ on Physalis peruviana. background were used ('Desiree' and 'Maris Bard'); in addition, two North American cultivars known to be susceptible to a wide range of PVY strains, 'Russet Burbank' and 'Russet Norkotah' (Rowley et al. 2015), were used for inoculations. These potato indicators were maintained as virus-free tissue-culture lines at the Potato Tissue Culture Laboratory at the UI and inoculated mechanically at the 6- to 10-leaf stage (Rowley et al. 2015). All plants were grown in an insectproof greenhouse before and after inoculations, under artificial light provided by fluorescent and incandescent lamps with a cycle of $18 \mathrm{~h}$ of day and $6 \mathrm{~h}$ of night, and maintained at 23 to $26^{\circ} \mathrm{C}$. The symptom observations started 4 to 5 days after inoculation and were carried out for 6 to 8 weeks. Three plants per species or cultivar were tested in a single experiment that always included the six poha isolates and control isolates, plus healthy control plants, and the experiments were repeated three times. Each infected plant was tested at 2 weeks postinoculation (wpi) (tobacco, pepper, and N. benthamiana) or 4 wpi (potato) for systemic infection and to confirm the identity of the inoculated PVY strain using triple-antibody sandwich enzyme-linked immunosorbent assay (TAS-ELISA) and RT-PCR, as described below.

Serological analysis, RT-PCR, and differentiating primers. The serological reactivity of the six poha isolates of PVY was tested in a TAS-ELISA format, as described by Nikolaeva et al. (2012). All tests included control PVY isolates from the laboratory collection, with distinct serological patterns characteristic of $\mathrm{PVYO}, \mathrm{PVY}^{\mathrm{N}}$, and $\mathrm{PVY} \mathrm{O}_{-} \mathrm{O} 5$ strains. In addition to a polyclonal antiserum (Funke et al. 2017; Karasev et al. 2010), three strain-specific monoclonal antibodies were used: Scottish Agricultural Science Agency (SASA)-O, recognizing PVYO, $\mathrm{PVYO}^{\mathrm{O}} \mathrm{O} 5, \mathrm{PVY}^{\mathrm{N}-\mathrm{W} / \mathrm{N}: \mathrm{O}}$, and PVYC; 1F5 (Agdia), recognizing PVYN, PVYO-O5, and PVYNTN; and SASA-N, recognizing PVYN and PVYNTN.

Two different multiplex RT-PCR assays were performed on the PVY isolates from poha, following the methods of Lorenzen et al. (2006) and Chikh-Ali et al. (2013). RT and PCR assays were performed essentially as described previously (Chikh-Ali et al. 2013, 2016). Control strains of PVY were obtained from the UI laboratory collection, and assays were conducted using the immunocapture (IC)-RT-PCR format (Chikh-Ali et al. 2013) on infected samples.

Sequencing and sequence analysis. The nearly whole-genome sequences of PVY isolates Poha1 through Poha6, missing only 65 to 85 nucleotides ( $\mathrm{nt}$ ) at the $5^{\prime}$ end due to the sequencing methodology used, were obtained by sequencing overlapping RT-PCR products, as described by Green et al. (2017). All PCR products were purified using the ExoSAP-IT for PCR Product Clean-Up (USB Products; Affymetrix Inc.), mixed with appropriate primers and submitted for Sanger sequencing to Genewiz, Inc. The contigs obtained were assembled using the SeqMan Pro program of the Lasergene 9 Suite (DNASTAR). For the multiple sequence alignments, MUSCLE (Edgar 2004) implemented in the program MEGA version 6 (Tamura et al. 2013) was used with the default parameters. The alignment consisted of all of the isolates listed in Supplementary Table S1. Sequence identity was checked using the BLAST program provided by the National Center for Biotechnology Information (Altschul et al. 1990). The aligned sequences were checked for recombination using the RDP (Martin and Rybicki 2000), GENECONV (Sawyer 1999), BOOTSCAN (Salminen et al. 1995), MAXCHI (Maynard-Smith, 1992), CHIMAERA (Posada and Crandall 2001), 3Seq (Boni et al. 2007), and SiScan (Gibbs et al. 2000) methods in RDP4 v4.61 software (Martin et al. 2005). Phylogeny inference was conducted based on the whole genomes using the maximum-likelihood (ML) method implemented in RDP v.4.61 (Martin et al. 2005). Bidens mosaic virus sequence KF649336 (Sanches et al. 2014) was used as an outgroup. Strain diversity within and between all nonrecombinant strains was calculated in MEGA 6 (Tamura et al. 2013) as given by $d=$ $\tau T-\frac{q}{q-1} \sum_{i-1}^{q} \sum_{j-1}^{q} x_{i} x_{j} d_{i j}$, where $d$ is the diversity, $x_{i}$ is the estimate of the average frequency of the $i$ th allele in the entire population, and $q$ is the number of different sequences in the entire sample (Tamura et al. 2013). The sequences were assigned to the appropriate strain "group", 
and the "Find best protein/DNA models (ML)" function was used with the default parameters to find the best model for the appropriate subset (group) of PVY sequences. Next, these models were used to change the appropriate settings of the "Compute mean diversity" function of MEGA 6 (Tamura et al. 2013).

\section{RESULTS}

PVY in Hawaiian poha. The attribution of the virus-like symptoms observed in poha plants grown on the island of Hawaii as associated with the presence of PVY occurred in 2014 through the use of RT-PCR with generic potyvirus primers and subsequent sequencing of a small, 300-nt genome fragment from the cytoplasmic inclusion (CI) cistron (not shown). Following this initial attribution, a very small survey of PVY presence in Hawaiian poha was conducted on the island of Hawaii in June 2015. Twenty-one symptomatic poha samples were collected from two locations, $1.6 \mathrm{~km}$ apart. One location represented conservation land, and six plants collected there grew wild; another location represented a commercial poha farm, where 15 samples were collected. All 21 samples collected in these two locations tested positive for PVY based on the general PVY immunostrip test. In August 2015, six symptomatic poha samples were collected at the commercial poha farm sampled 2 months earlier and sent to the UI laboratory for further studies. At UI, these six poha samples were confirmed to be infected with PVY using TAS-ELISA; they were randomly assigned numbers, from Poha1 to Poha6, inoculated to tobacco, and, upon confirmation of systemic infection, maintained as part of the laboratory collection.

Biological characterization of PVY from poha in four solanaceous species. Four species of Solanaceae were tested for susceptibility to and symptom expression from the poha PVY infection: tobacco, pepper, $N$. benthamiana, and four cultivars of potato (Table 1). Tobacco and $N$. benthamiana were found to be susceptible to poha PVY isolates, developing a systemic infection within $2 \mathrm{wpi}$, and the virus was readily detectable by ELISA and RT-PCR. In tobacco, all six PVY isolates induced mild symptoms of mosaic and vein clearing at $2 \mathrm{wpi}$, similar to the symptoms induced by the control isolates H14, AL100001, and NY110001 (all three PVYC), and also by isolate Oz (PVYO). Another control isolate, N605 (PVYN), induced vein necrosis in tobacco but was unable to infect pepper (Table 1). All six poha PVY isolates induced severe systemic necrosis in $N$. benthamiana, leading to plant death within 3 to 4 wpi (Table 1), similar to symptoms induced by three control PVYC isolates $\mathrm{H} 14$, AL100001, and NY110001 and in contrast to both Oz (PVYO) and N605 (PVYN), which induced mosaic and stunting in infected plants but no necrosis or death (Table 1).

In C. annuum Early California Wonder pepper, all six poha PVY isolates induced no visible symptoms, whereas the control isolates
H14 (PVYC), AL100001 (PVYC), and PVY-Oz (PVYO) induced mild mosaic. One control isolate, NY110001 (PVYC), induced systemic vein necrosis in pepper (Table 1). No systemic infection was produced by any of the six poha PVY isolates in pepper as determined by the testing of the upper, uninoculated leaves using ELISA at 2 to 4 wpi. Nevertheless, at least three poha isolates (Poha2, Poha3, and Poha6) were found replicating in inoculated leaves of pepper plants at $4 \mathrm{wpi}$ as determined by ELISA (Table 1). Another control isolate, N605 $\left(\mathrm{PVY}^{\mathrm{N}}\right)$, was unable to infect pepper systemically, as was expected (Chikh-Ali et al. 2016; Moury 2010), although virus replication was detectable in inoculated leaves (Table 1). The lack of systemic infection in pepper suggested that poha PVY isolates may belong to the potato PVY strains.

In potato, however, only isolates Poha2 and Poha5 induced necrotic lesions on inoculated leaves in Maris Bard, visible at 2 wpi (Fig. 2A), suggesting triggering of the $N c_{t b r}$ gene because no necrotic lesions were induced in Desiree in the same experiment (Fig. 2B). Four of the six poha PVY isolates (Poha1, Poha2, Poha3, and Poha5) induced necrotic lesions on inoculated leaves in Russet Burbank and Russet Norkotah (Fig. 3A and B). All six poha PVY isolates were able to replicate in inoculated leaves of all four tested potato cultivars, as determined by ELISA testing at 2 to 3 wpi, but no systemic infection was detected for any of the six poha PVY isolates in any of the four tested cultivars (Table 1). This was similar to the reactions in potato cultivars to three PVYC controls (H14, AL100001, and NY110001), where none of them was able to systemically infect the tested potato cultivars, although all three induced the hypersensitive resistance response (HR) in inoculated leaves of Maris Bard (Table 1). One of these PVYC controls, AL100001, also induced HR in inoculated leaves of Desiree and Russet Norkotah, while NY110001 induced $\mathrm{HR}$ in inoculated leaves of Desiree in addition to Maris Bard (Table 1). It contrasted with PVY isolate $\mathrm{Oz}(\mathrm{PVYO})$ that induced $\mathrm{HR}$ in Desiree and Maris Bard (Fig. 2) and systemically infected Russet Burbank and Russet Norkotah, and with N605 (PVYN) that systemically infected all four cultivars (Table 1), as expected (Moury 2010; Singh et al. 2008). The inability of the poha PVY isolates to systemically infect both pepper and potato suggested an unusual combination of biological properties, and prompted further study of the six isolates using molecular tools.

Serological and molecular typing. Serological typing of the six poha PVY isolates is summarized in Table 2. All six poha PVY isolates reacted with the strain-nonspecific antibody Asc6 (Funke et al. 2017), while three of them (Poha1, Poha2, and Poha3) reacted with the O-specific antibody SASA-O provided by the commercial supplier (SASA). None of the six poha PVY isolates bound, however, two N-specific antibodies, 1F5 and SASA-N, provided by Agdia and SASA, respectively (Table 2). Based on these ELISA data, isolates

TABLE 1. Symptoms induced by six poha isolates of Potato virus $Y$ (PVY), Poha1 to Poha6, plus control isolates, in different indicator plants ${ }^{\mathrm{a}}$

\begin{tabular}{|c|c|c|c|c|c|c|c|}
\hline \multirow[b]{2}{*}{ PVY isolate } & \multirow[b]{2}{*}{ Nicotiana benthamiana } & \multirow[b]{2}{*}{ Tobacco $^{\mathrm{b}}$} & \multirow[b]{2}{*}{ Pepper ${ }^{\mathrm{c}}$} & \multicolumn{4}{|c|}{ Potato } \\
\hline & & & & Russet Burbank & Russet Norkotah & Desiree & Maris Bard \\
\hline Poha1 & nec, syst HR, st, death & $\mathrm{mo}, \mathrm{vcl}$ & $\mathrm{NS}$, ni & HR, loi, NS, ni & HR, loi, NS, ni & loi, NS, ni & loi, NS, ni \\
\hline Poha2 & nec, syst HR, st, death & $\mathrm{mo}, \mathrm{vcl}$ & loi, NS, ni & HR, loi, NS, ni & HR, loi, NS, ni & loi, NS, ni & HR, loi, NS, ni \\
\hline Poha3 & nec, syst HR, st, death & mo, vcl & loi, NS, ni & HR, loi, NS, ni & HR, loi, NS, ni & HR, loi, NS, ni & loi, NS, ni \\
\hline Poha4 & nec, syst HR, st, death & mo, vcl & NS, ni & loi, NS, ni & loi, NS, ni & loi, NS, ni & loi, NS, ni \\
\hline Poha5 & nec, syst HR, st, death & $\mathrm{mo}, \mathrm{vcl}$ & NS, ni & HR, loi, NS, ni & HR, loi, NS, ni & loi, NS, ni & HR, loi, NS, ni \\
\hline Poha6 & nec, syst HR, st, death & $\mathrm{mo}, \mathrm{vcl}$ & loi, NS, ni & loi, NS, ni & loi, NS, ni & loi, NS, ni & loi, NS, ni \\
\hline H14 & nec, syst HR, st, death & $\mathrm{mo}, \mathrm{vcl}$ & mo, mtl & NT & $\mathrm{NS}$, ni & $\mathrm{NS}$, ni & loi, NS, ni \\
\hline AL100001 & nec, syst HR, st, death & $\mathrm{mo}, \mathrm{vcl}$ & mo, mtl & NT & loi, NS, ni & loi, NS, ni & loi, NS, ni \\
\hline NY110001 & nec, syst HR, st, death & $\mathrm{mo}, \mathrm{vcl}$ & $\mathrm{vn}$ & NT & NS, ni & loi, NS, ni & loi, NS, ni \\
\hline & $\mathrm{mo}, \mathrm{mtl}, \mathrm{st}$ & $\mathrm{mo}, \mathrm{vcl}$ & mo, mtl & mo, mtl & mo, mtl & $\mathrm{HR}, \mathrm{mo}, \mathrm{mtl}$ & $\mathrm{HR}, \mathrm{mo}, \mathrm{mtl}$ \\
\hline N605 & $\mathrm{mo}, \mathrm{mtl}, \mathrm{st}$ & vn & loi, NS, ni & NS & mo & NS & NS \\
\hline
\end{tabular}

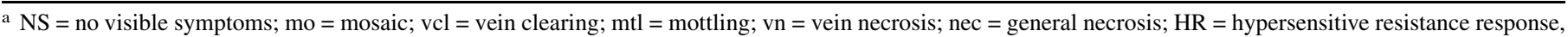
local necrotic lesions on inoculated leaves; syst HR = systemic HR; st = stunting; loi = local virus infection in an inoculated leaf as determined by triple-antibody sandwich enzyme-linked immunosorbent assay (TAS-ELISA); ni = no systemic infection in upper, uninoculated leaves as determined by TAS-ELISA; and NT = not tested.

b Burley.

c Early California Wonder. 
Poha1, Poha2, and Poha3 appeared to have an O serotype, similar to two PVYC controls (H14 and AL100001), whereas isolates Poha4, Poha5, and Poha6 appeared to have no identifiable serotype, similar to another PVYC control (NY110001) (Table 2).

When subjected to the RT-PCR typing with the multiplex assay developed by Lorenzen et al. (2006), all six poha PVY isolates produced only one 267-bp band indicative of the presence of the PVYO sequence, similar to the three PVYC controls H14, AL100001, and NY110001 (Table 3). Thus, poha PVY isolates were clearly different from PVYN isolate N605, producing characteristic bands of 328 and 398 bp (Table 3). However, a true PVYO sequence also produces the 689-bp band, like the PVY-Oz isolate (Table 3). The second RT-PCR typing assay (Chikh-Ali et al. 2013) produced approximately 500-bp bands in all six poha PVY isolates tested (Table 3). This result appeared inconclusive because no specific band of this size is associated with any of the known recombinant or nonrecombinant types of PVY (Chikh-Ali et al. 2013). The three PVYC controls (H14, AL100001, and NY110001) produced no bands in the assay of Chikh-Ali et al. (2013). The control isolate Oz (PVYO) produced two characteristic bands in this assay (532 and $853 \mathrm{bp}$ ) while isolate N605 $\left(\mathrm{PVY}^{\mathrm{N}}\right)$ produced three characteristic bands (398, 633 , and 1,307 bp) (Table 3). The combination of a unique biological profile, $\mathrm{O}$ (Poha1, Poha2, and Poha3) or uncertain (Poha4, Poha5, and Poha6) serology, and unusual molecular typing results for the six poha PVY isolates suggested a set of novel sequences, distinct from the currently known PVY genotypes.

Whole-genome sequences. The nearly complete, approximately 9.6-kb genomes of the poha PVY isolates Poha1 to Poha6 were determined through the Sanger sequencing of overlapping RTPCR fragments and deposited in the GenBank database under the accession numbers MF134861 to MF134866. A 3,061-amino-acid polyprotein was encoded by a single open reading frame (ORF) in each of the six sequences. This polyprotein was found to encode 10 cistrons typical of PVY and other potyviruses, including the capsid protein $(\mathrm{CP})$ at its $\mathrm{C}$ terminus. The $\mathrm{CP}$ had the "DAG" motif at the

TABLE 2. Serological reactivity of the six poha Potato virus $Y$ (PVY) isolates, marked Poha1 through Poha6, and two control isolates with different monoclonal antibodies tested in triple-antibody sandwich enzyme-linked immunosorbent assay (TAS-ELISA)

\begin{tabular}{lcccc}
\hline & \multicolumn{5}{c}{ TAS-ELISA } \\
\cline { 2 - 5 } Isolates (strain) & Asc6 $^{\mathrm{a}}$ & SASA-O $^{\mathrm{b}}$ & 1F5 $^{\mathrm{b}}$ & SASA-N $^{\mathrm{b}}$ \\
\hline Poha1 & + & + & - & - \\
Poha2 & + & + & - & - \\
Poha3 & + & + & - & - \\
Poha4 & + & - & - & - \\
Poha5 & + & - & - & - \\
Poha6 & + & - & - & - \\
H14 & + & + & - & - \\
AL100001 & + & + & - & - \\
NY110001 & + & - & - & - \\
Oz (O) & + & + & - & - \\
N605 (N) & + & - & + & + \\
\hline
\end{tabular}

a Polyclonal antibody: Asc6 reacts with all PVY strains (Funke et al. 2017).

b Monoclonal antibodies: SASA-O specific to PVYO or PVYC 1 F5 specific to PVYO-O5 or PVYN/NTN and SASA-N specific to PVYN/NTN.

\section{A Maris Bard}
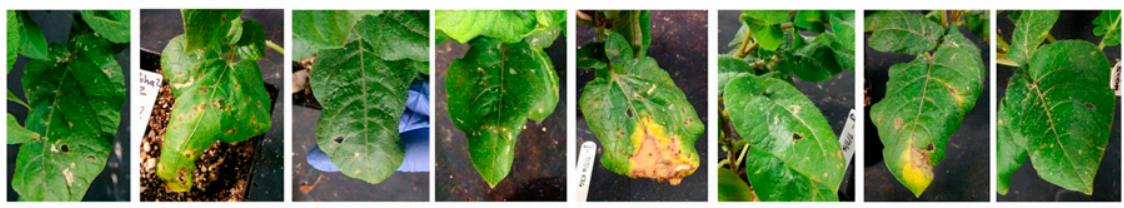

B Desiree

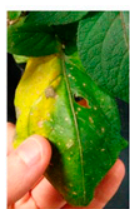

Poha1

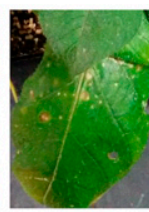

Poha2

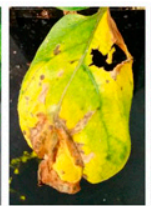

Poha3

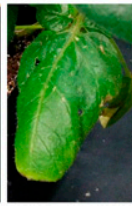

Poha4

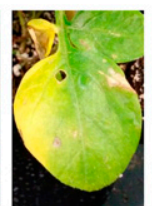

Poha5

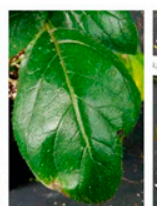

Poha6

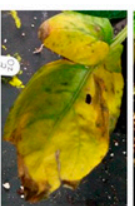

$\mathrm{Oz}$

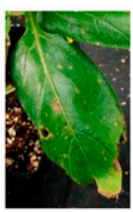

N605

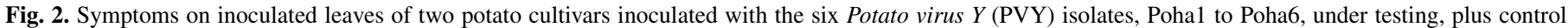
isolates $\mathrm{Oz}\left(\mathrm{PVY}^{\mathrm{O}}\right)$ and N605 $\left(\mathrm{PVY}^{\mathrm{N}}\right)$. A, Maris Bard, 21 days postinoculation (dpi) and B, Desiree, $18 \mathrm{dpi}$.

\section{A Russet Norkotah}
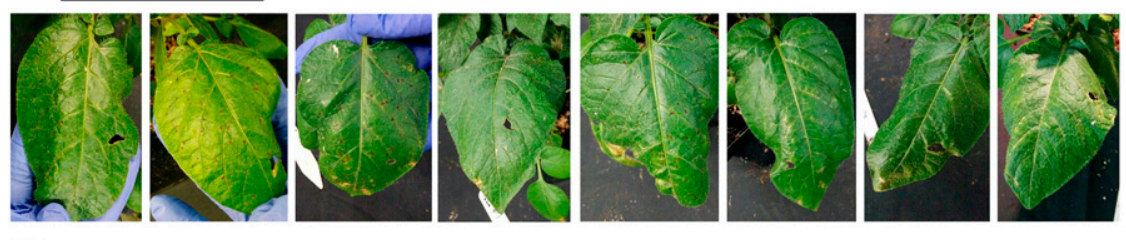

B Russet Burbank

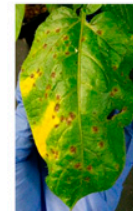

Poha1

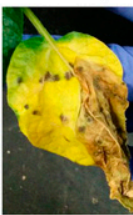

Poha2

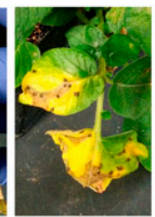

Poha3

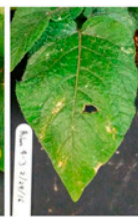

Poha4

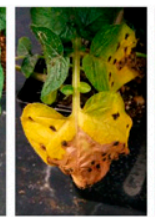

Poha5

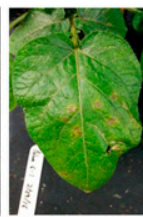

Poha6

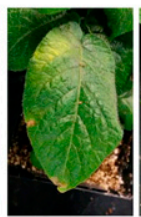

$\mathrm{Oz}$

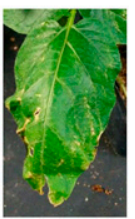

N605

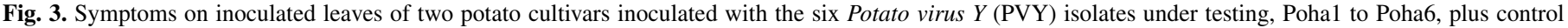
isolates $\mathrm{Oz}\left(\mathrm{PVY}^{\mathrm{O}}\right)$ and $\mathrm{N} 605\left(\mathrm{PVY}^{\mathrm{N}}\right)$. A, Russet Norkotah, 10 days postinoculation (dpi) and B, Russet Burbank, 21 dpi. 
$\mathrm{N}$ terminus associated with aphid transmission of potyviruses (Atreya et al. 1991). An out-of-frame, small ORF coding for the P3N-PIPO protein characteristic of potyviruses (Chung et al. 2008; Wei et al. 2010) was present in all six poha PVY genomes; these P3N-PIPO proteins were $100 \%$ identical in amino acid sequence among themselves (Supplementary Fig. S1). Sequence identity levels between the whole genomes of the six poha PVY isolates differed, ranging from 99\% between Poha4 and Poha6 to $96 \%$ between Poha 2 and Poha4.

When subjected to a BLASTN search through the GenBank database (Altschul et al. 1990), all six poha PVY sequences were closest in identity (91\%) to the sequence of the PRI-509 isolate (GenBank accession EU563512) belonging to the PVYC strain of PVY (Dullemans et al. 2011). In phylogenetic reconstructions conducted with 32 nonrecombinant whole-genome sequences representing

TABLE 3. Reverse-transcription polymerase chain reaction (RT-PCR)-based strain typing of the six poha Potato virus $Y$ (PVY) isolates, labeled Poha1 through Poha6, using two multiplex RT-PCR assays, in comparison with the two control isolates, $\mathrm{Oz}\left(\mathrm{PVY}^{\mathrm{O}}\right)$ and $\mathrm{N} 605\left(\mathrm{PVY}^{\mathrm{N}}\right)$

\begin{tabular}{|c|c|c|c|}
\hline \multirow[b]{2}{*}{ PVY isolate } & \multicolumn{2}{|c|}{ Multiplex bands } & \multirow[b]{2}{*}{ Strain typing results } \\
\hline & PCR $1(b p)^{a}$ & $\operatorname{PCR} 2(b p)^{\mathrm{a}}$ & \\
\hline Poha1 & $267 \mathrm{bp}$ & Approximately 500 bp & C (?) \\
\hline Poha2 & $267 \mathrm{bp}$ & Approximately 500 bp & C (?) \\
\hline Poha3 & $267 \mathrm{bp}$ & Approximately 500 bp & C (?) \\
\hline Poha4 & $267 \mathrm{bp}$ & Approximately 500 bp & C (?) \\
\hline Poha5 & $267 \mathrm{bp}$ & Approximately 500 bp & C (?) \\
\hline Poha6 & $267 \mathrm{bp}$ & Approximately 500 bp & $\mathrm{C}(?)$ \\
\hline $\mathrm{H} 14$ & $267 \mathrm{bp}$ & No bands & $\mathrm{C}$ \\
\hline AL100001 & $267 \mathrm{bp}$ & No bands & $\mathrm{C}$ \\
\hline NY110001 & $267 \mathrm{bp}$ & No bands & $\mathrm{C}$ \\
\hline $\mathrm{Oz}^{\mathrm{c}}$ & $267+689$ bp & $532+853$ bp & $\mathrm{O}$ \\
\hline $\mathrm{N} 605^{\mathrm{c}}$ & $328+398 b p$ & $398+633+1,307 \mathrm{bp}$ & $\mathrm{N}$ \\
\hline
\end{tabular}

${ }^{a}$ PCR 1 according to Lorenzen et al. (2006).

b PCR 2 according to Chikh-Ali et al. (2013)

${ }^{c} \mathrm{Oz}$ and $\mathrm{N} 605$ are reference isolates of $\mathrm{PVY}$ strains $\mathrm{PVY}^{\mathrm{O}}$ and $\mathrm{PVY}^{\mathrm{N}}$, respectively, maintained in the PVY isolate collection of the University of Idaho. all major strains of PVY, all six poha PVY isolates were placed within the diverse PVYC lineage and grouped together as a phylogenetically monophyletic clade clearly separate from other PVYC clades which included PVY isolates from pepper, tomato, potato, or tobacco (Fig. 4). Poha PVY sequences in this phylogenetic analysis were found to be quite distinct from sequences of the $\mathrm{C} 1$ and $\mathrm{C} 2$ clades, and formed a new Poha clade (Fig. 4). No evidence of recombination was found when these six poha PVY sequences were analyzed using the programs from the RDP4 suite (not shown), whereas two of the control PVYC isolates, AL100001 and NY110001, were easily identified as recombinants containing small segments of NE-11 and PVYO sequences, respectively, in their CI cistrons (Green et al. 2017). Because of their recombinant nature, neither AL100001 nor NY110001 was included in the phylogenetic analysis presented in Figure 4.

Because all six poha PVY sequences were placed in a single clade with long branches, we estimated the genetic diversity of this Poha clade of PVY sequences relative to genetic diversity of other PVY clades such as large clades combining $\mathrm{PVY}^{\mathrm{O}}, \mathrm{PVY}^{\mathrm{N}}$, and $\mathrm{PVYC}$, plus individual subclades of PVYO, PVYO-O5, C1, C2, PVYEu-N, and PVYNA-N (Table 4); the model details for each subset are listed in Table 4. Genetic diversity of the Poha clade was $54 \%$ higher than the diversity in the entire PVYN lineage and $82 \%$ higher than in the entire PVYO lineage (Table 4). Diversity in individual subclades of PVYO, PVYO-O5, and PVYEu-N appeared to be 3.1 to 5.7 times lower than in the Poha clade, whereas the more diverse PVYNA-N subclade showed diversity 1.8 times lower than in the Poha clade (Table 4).

\section{DISCUSSION}

Biological properties of the six poha isolates of PVY collected on the island of Hawaii were found distinct from another PVYC isolate, PVY-H14, collected on the island of Oahu from tomato (Chikh-Ali et al. 2016). Unlike the PVY-H14 isolate, the poha PVY isolates were unable to systemically infect pepper (Table 1); however, just like PVYH14, all six poha PVY isolates could not systemically infect potato (Table 1). Clearly, different populations of PVYC isolates from distinct evolutionary clades can be found in the Hawaiian Islands, affecting

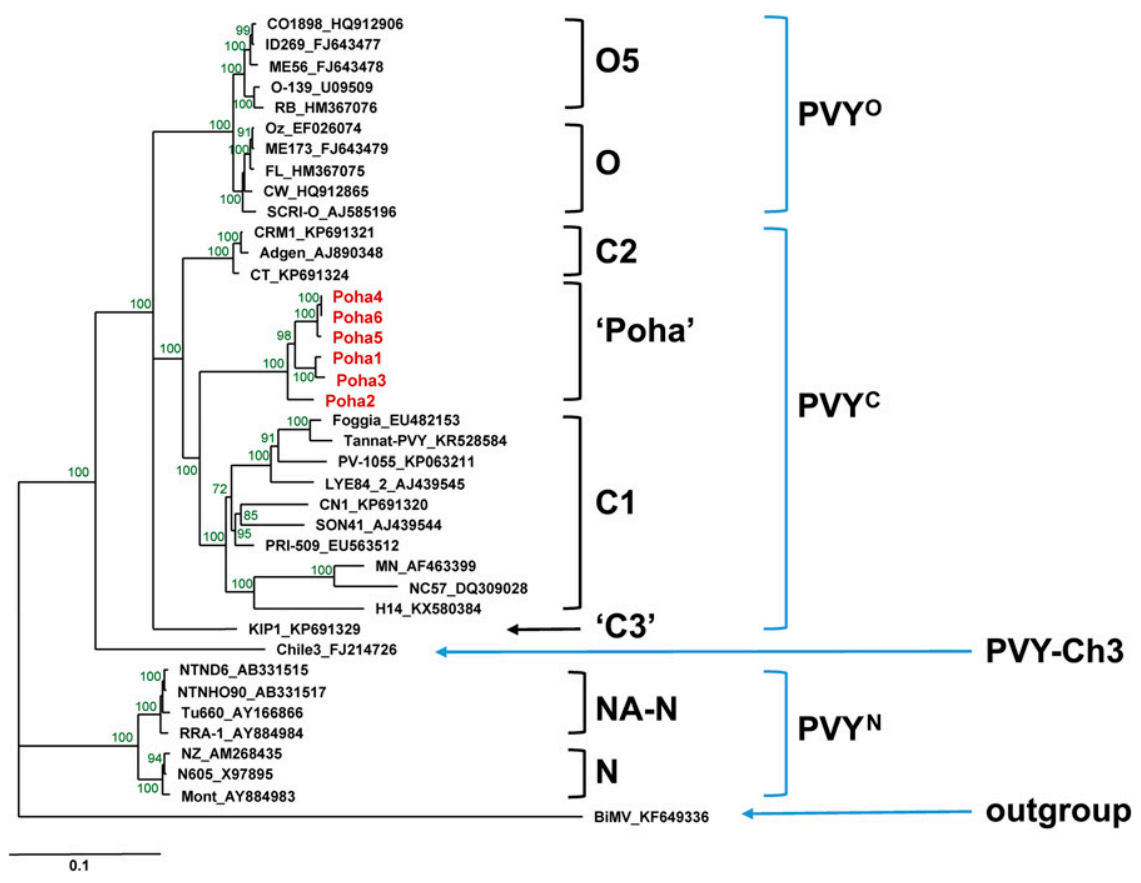

Fig. 4. Maximum-likelihood phylogenetic tree for the full genomes of nonrecombinant Potato virus $Y$ (PVY) isolates. Sequence alignment was done using MUSCLE provided in MEGA 6, and the tree was drawn using the RDP 4 v4.61 program, for PVY isolates with no detected recombination. Bootstrap values of $70 \%$ or higher are shown at the corresponding nodes. Abbreviations of the PVY isolate names are followed by the corresponding GenBank accessions. Blue brackets indicate main lineages of $\mathrm{PVY}^{\mathrm{O}}, \mathrm{PVY}^{\mathrm{C}}, \mathrm{PVY}^{\mathrm{N}}$, and Chile 3, associated with strain groups, while black brackets indicate additional lineages within main PVY strain groups. The Bidens mosaic virus (BiMV) sequence was used as an outgroup. 
different crops such as tomato and poha ( $P$. peruviana). Two other PVYC controls used here are recombinants carrying small pieces of PVYO (NY110001) or PVY-NE11 (AL100001) sequences in their CI cistron (Green et al. 2017), and they also differed from the poha PVY isolates in their ability to systemically infect pepper (Table 1).

The inability of the six poha PVY isolates to systemically infect potato did not prevent replication of all six in the inoculated leaves, suggesting unimpaired cell-to-cell movement of the virus in potato. Three of the six poha isolates were also able to move cell to cell in inoculated leaves in pepper. Apparently, potato and pepper cultivars tested exhibited resistance to poha PVY (Table 1) which, in the case of potato, could be related to the expression of an HR visible as local necrotic lesions on inoculated leaves (Figs. 2 and 3). The Poha2 and Poha5 isolates elicited HR in Maris Bard but not in Desiree (Fig. 2) - this may be due to the triggering of the $N c_{t b r}$ gene present in Maris Bard but not in Desiree (Cockerham 1970; Jones 1990) because both isolates belonged to the PVYC strain. However, the other four isolates (Poha1, Poha3, Poha4, and Poha6) did not elicit any visible signs of $\mathrm{HR}$ in inoculated leaves of Maris Bard and Desiree (Fig. 2), suggesting that reliance on the triggering of $N c_{t b r}$ for classifying PVYC isolates may not be very useful. This conclusion is consistent with the HR elicited in Maris Bard by the three PVYC controls, suggesting triggering of the $N c_{t b r}$ but also eliciting HR in Desiree by two of them (NY110001 and AL100001), whereas the presence of the $N c_{t b r}$ is not expected in Desiree (Table 1). Interestingly, four of the six poha isolates of PVY (Poha1, Poha2, Poha3, and Poha5) elicited HR in two North American cultivars of potato (Russet Norkotah and Russet Burbank) (Fig. 3) that are not known to have any resistance against PVY strains commonly found in potato (Rowley et al. 2015). One of the PVYC controls, AL100001, also elicited HR in Russet Norkotah (Table 1). The observed HR-like reaction on inoculated leaves of Russet Burbank and Russet Norkotah inoculated with four of the poha PVY isolates (Fig. 3) may indicate the presence of a corresponding resistance allele in their genetic background (Kehoe and Jones 2016), which phenotypically restricted systemic movement of these poha isolates of PVY in potato.

Up to now, all isolates of PVY could be classified as either potato or pepper adapted, and the phylogenies of PVY strains grouped potato isolates mainly into $\mathrm{PVYO}^{\mathrm{O}}$ or $\mathrm{PVY}^{\mathrm{N}}$ lineages and pepper isolates into the PVYC or Chile3 lineages (Chikh-Ali et al. 2016; Dullemans et al. 2011; Moury 2010; Quenouille et al. 2013; Singh et al. 2008). Nevertheless, the PVYC lineage contained a number of potato isolates of PVY (Blanco-Urgoiti et al. 1998; Dullemans et al. 2011; Kehoe and Jones 2016), and this potato-pepper distinction was not strict. The obvious diversity of the PVYC lineage (ChikhAli et al. 2016; Dullemans et al. 2011; Moury 2010; Quenouille et al. 2013) (Table 4) suggested that it was not yet studied enough to reveal any firm relationships between PVYC phylogenies and host specialization. The data also suggested that other solanaceous hosts, distinct from potato or pepper, may be involved in $\mathrm{PVYC}$ phylogeny and evolution, such as poha (P. peruviana) (Fig. 4).

The six poha isolates of PVY formed a separate clade within the PVYC lineage (Fig. 4), clearly distinguished from $\mathrm{C} 1$ and $\mathrm{C} 2$ clades

TABLE 4. Genetic diversity within and between clades of Potato virus $Y$ (PVY) strains, calculated as described in Materials and Methods ${ }^{\mathrm{a}}$

\begin{tabular}{lcclcc}
\hline Clades & Diversity & Standard error & Model & $\mathrm{I}$ & $\mathrm{G}$ \\
\hline $\mathrm{O}+\mathrm{O} 5$ & 0.022 & 0.001 & $\mathrm{TN} 93+\mathrm{G}$ & $\mathrm{n} / \mathrm{a}$ & 0.29 \\
$\mathrm{O}$ & 0.011 & 0.001 & TN93 & $\mathrm{n} / \mathrm{a}$ & $\mathrm{n} / \mathrm{a}$ \\
$\mathrm{O} 5$ & 0.013 & 0.001 & TN93 & $\mathrm{n} / \mathrm{a}$ & $\mathrm{n} / \mathrm{a}$ \\
Eu-N + NA-N & 0.026 & 0.001 & TN93+G & n/a & 0.45 \\
Eu-N & 0.007 & 0.000 & HKY & n/a & n/a \\
NA-N & 0.022 & 0.001 & TN93+G & n/a & 0.28 \\
C (excluding Poha) & 0.151 & 0.005 & TN93+G & n/a & 0.27 \\
Poha & 0.040 & 0.002 & TN93+G & n/a & 0.09 \\
All & 0.181 & 0.003 & GTR+I+G & 0.42 & 1.23 \\
\hline
\end{tabular}

a Strains are defined as in Karasev and Gray (2013); n/a = not available. established earlier (Blanco-Urgoiti et al. 1998; Dullemans et al. 2011; Moury 2010). The original separation of the $C 1$ and $C 2$ clades was based on CP gene sequences for PVY isolates placed in the PVYC lineage (Blanco-Urgoiti et al. 1998) and included many partial sequences. Separation of these two clades held up when the analysis was conducted on a smaller number of whole-genome sequences as well (Dullemans et al. 2011; Kehoe and Jones 2016; Moury 2010; Quenouille et al. 2013). Addition of six PVY isolates from poha to the phylogenetic analysis reinforced this separation but also added the Poha clade and perhaps an additional C3 clade (Fig. 4), represented thus far by a single sequence of the KIP1 isolate originally from potato (Kehoe and Jones 2016). Interestingly, both clades C2 and C3 include only potato PVY isolates, while the diverse clade $\mathrm{C} 1$ includes $\mathrm{PVY}$ isolates from potato, pepper, tomato, and other hosts (Fig. 4).

Of the four main evolutionary lineages distinguished in PVY (Chikh-Ali et al. 2016; Moury 2010; Quenouille et al. 2013), the PVYC lineage is the most diverse, both biologically and evolutionarily (Fig. 4; Table 4). Indeed, it is the only lineage combining PVY isolates from pepper, potato, tomato, poha, and other hosts (Fig. 4) (Chikh-Ali et al. 2016; Green et al. 2017), whereas PVYN and PVYO lineages include only isolates from potato (Green et al. 2017) and the Chile 3 lineage consists of only one PVY isolate from pepper (Moury 2010). In addition to including PVY isolates from multiple hosts, the PVYC lineage is also the most diverse lineage evolutionarily (Table 4), exhibiting a diversity level similar to the diversity of the entire PVY species. The entire PVYC clade, excluding the six poha sequences, exhibited diversity of only $20 \%$ lower than all the PVY sequences taken together (Table 4), suggesting that the PVYC clade currently defined as PVYC strain (Quenouille et al. 2013; Singh et al. 2008) may need separation into additional strains. This apparent diversity of the PVYC lineage may be interpreted as an indication of an ancestral position of this lineage relative to PVYN, $\mathrm{PVYO}$, and perhaps even Chile3. Although only one whole-genome sequence is currently available for the Chile3 lineage, two other PVY-positive samples collected from the same host, Chile1 and Chile2, were sequenced partially (Moury 2010) and both exhibited very high identity levels to the Chile3 sequence ( $99 \%$ ), which may be consistent with the low diversity level in the Chile 3 lineage.

Poha (P. peruviana) was introduced to Hawaii in the early 19 th century (National Academy of Sciences 1989) and, since then, has been cultivated there for approximately 200 years. However, the degree of the genetic diversity of the PVYC Poha clade (Table 4; Fig. 4) may suggest that PVY and $P$. peruviana have been associated with each other at least as long as other potato clades (PVYO and PVYN) have been associated with potato. Given the widespread cultivation of $P$. peruviana across the globe, it would be interesting to determine the possible evolutionary links of PVY isolates from this host species from other geographical areas, distant from Hawaii. An alternative scenario would be an as-yet-unknown species associated with these poha PVY isolates in Hawaii and serving as an inoculum source for the poha crop. In this case, poha plants would be considered merely indicators of the PVY infection. To determine which of the two scenarios explains the presence of these new poha PVY isolates, additional surveys of plants on the island of Hawaii may be needed.

\section{ACKNOWLEDGMENTS}

We thank L. Ewing and J. Durrin for providing potato tissue plantlets, J. Chojnacky and C. Funke for isolate collection maintenance and laboratory and greenhouse help, and S. Motomura for sample collection. This work was funded, in part, through grants from the United States Department of Agriculture (USDA) National Institute of Food and Agriculture (NIFA) National Research Initiative (2009-35600-05025), USDA-NIFA Specialty Crops Research Initiative (2014-51181-22373), USDA-NIFA-Hatch (project IDA0560), USDA Agricultural Research Service (58-1907-3-015, 58-5352-4006, and 58-8042-6-049), Idaho State Department of Agriculture, Northwest Potato Research Consortium, Idaho Potato Commission, Washington State Potato Commission, and by the Idaho Agricultural Experiment Station. 


\section{LITERATURE CITED}

Adams, M. J., Zerbini, F. M., French, R., Rabenstein, F., Stenger, D. C., and Valkonen, J. P. T. 2012. Potyviridae. Pages 1069-1089 in: Virus Taxonomy. Ninth Report of the International Committee on Taxonomy of Viruses. A. M. Q. King, M. J. Adams, E. B. Carstens, and E. J. Lefkowitz, eds. Elsevier, Amsterdam.

Altschul, S. F., Gish, W., Miller, W., Myers, E. W., and Lipman, D. J. 1990. Basic local alignment search tool. J. Mol. Biol. 215:403-410.

Atreya, P. L., Atreya, C. D., and Pirone, T. P. 1991. Amino acid substitutions in the coat protein result in loss of insect transmissibility of a plant virus. Proc. Natl. Acad. Sci. USA 88:7887-7891.

Blanco-Urgoiti, B., Sanchez, F., Pérez de San Román, C., Dopazo, J., and Ponz, F. 1998. PVYC isolates are a homogeneous pathotype but two different genetic strains. J. Gen. Virol. 79:2037-2042.

Boni, M. F., Posada, D., and Feldman, M. W. 2007. An exact nonparametric method for inferring mosaic structure in sequence triplets. Genetics 176: 1035-1047.

Chikh-Ali, M., Gray, S. M., and Karasev, A. V. 2013. An improved multiplex IC-RT-PCR assay distinguishes nine strains of Potato virus Y. Plant Dis. 97: 1370-1374.

Chikh-Ali, M., Vander Pol, D., Nikolaeva, O. V., Melzer, M. J., and Karasev, A. V. 2016. Biological and molecular characterization of a tomato isolate of Potato virus $Y$ (PVY) of the PVYC lineage. Arch. Virol. 161:3561-3566.

Chung, B. Y., Miller, W. A., Atkins, J. F., and Firth, A. E. 2008. An overlapping essential gene in the Potyviridae. Proc. Natl. Acad. Sci. USA 105:5897-5902.

Cockerham, G. 1970. Genetic studies on resistance to potato viruses X and Y. Heredity 25:309-348.

Dullemans, A. M., Cuperus, C., Verbeek, M., and van der Vlugt, R. A. A. 2011. Complete nucleotide sequence of a potato isolate of strain group $\mathrm{C}$ of Potato virus $Y$ from 1938. Arch. Virol. 156:473-477.

Edgar, R. C. 2004. MUSCLE: Multiple sequence alignment with high accuracy and high throughput. Nucleic Acids Res. 32:1792-1797.

Funke, C. N., Nikolaeva, O. V., Green, K. J., Tran, L. T., Chikh-Ali, M., Quintero-Ferrer, A., Cating, R., Frost, K. E., Hamm, P. B., Olsen, N., Pavek, M. J., Gray, S. M., Crosslin, J. M., and Karasev, A. V. 2017. Strain-specific resistance to Potato virus $Y$ (PVY) in potato and its effect on the relative abundance of PVY strains in commercial potato fields. Plant Dis. 101:20-28.

Gibbs, M. J., Armstrong, J. S., and Gibbs, A. J. 2000. Sister-scanning: A Monte Carlo procedure for assessing signals in recombinant sequences. Bioinformatics 16:573-582.

Green, K. J., Brown, C. J., Gray, S. M., and Karasev, A. V. 2017. Phylogenetic study of recombinant strains of Potato virus Y. Virology 507:40-52.

Hamasaki, R. T., Motomura, S. A., Melzer, M. J., and Bushe, B. C. 2015. Potato virus $Y$ : A pathogen associated with an emerging disease of poha in Hawai'i. UH-CTAHR Ext. Publ. PD-109. University of Hawaii-Manoa, Honolulu, HI.

Hu, X., Meacham, T., Ewing, L., Gray, S. M., and Karasev, A. V. 2009. A novel recombinant strain of Potato virus $Y$ suggests a new viral genetic determinant of vein necrosis in tobacco. Virus Res. 143:68-76.

Jones, R. A. C. 1990. Strain group specific and virus specific hypersensitive reactions to infection with potyviruses in potato cultivars. Ann. Appl. Biol. 117:93-105.

Karasev, A. V., and Gray, S. M. 2013. Continuous and emerging challenges of Potato virus $Y$ in potato. Annu. Rev. Phytopathol. 51:571-586.
Karasev, A. V., Nikolaeva, O. V., Hu, X., Sielaff, Z., Whitworth, J., Lorenzen, J. H., and Gray, S. M. 2010. Serological properties of ordinary and necrotic isolates of Potato virus Y: A case study of PVY ${ }^{\mathrm{N}}$ misidentification. Am. J. Potato Res. 87:1-9.

Kehoe, M. A., and Jones, R. A. C. 2016. Improving Potato virus Y strain nomenclature: Lessons from comparing isolates obtained over a 73-year period. Plant Pathol. 65:322-333.

Kerlan, C. 2006. Potato virus Y. Descriptions of Plant Viruses, No 414. Association of Applied Biologists, Wellesbourne, U.K. www.dpvweb.net/dpv/ showdpv.php?dpvno=414

Lorenzen, J. H., Piche, L. M., Gudmestad, N. C., Meacham, T., and Shiel, P. 2006. A multiplex PCR assay to characterize Potato virus $Y$ isolates and identify strain mixtures. Plant Dis. 90:935-940.

Martin, D., and Rybicki, E. 2000. RDP: Detection of recombination amongst aligned sequences. Bioinformatics 16:562-563.

Martin, D., Williamson, C., and Posada, D. 2005. RDP2: Recombination detection and analysis from sequence alignment. Bioinformatics 21:260-262.

Maynard-Smith, J. 1992. Analyzing the mosaic structure of genes. J. Mol. Evol. 34:126-129.

Moury, B. 2010. A new lineage sheds light on the evolutionary history of Potato virus Y. Mol. Plant Pathol. 11:161-168.

National Academy of Sciences. 1989. Pages 241-252 in: Lost Crops of the Incas: Little-Known Plants of the Andes with Promise for Worldwide Cultivation. National Academies Press, Washington, DC.

Nikolaeva, O. V., Roop, D., Galvino-Costa, S. F. B., Figueira, A. R., Gray, S. M., and Karasev, A. V. 2012. Epitope mapping for monoclonal antibodies recognizing tuber necrotic strains of Potato virus Y. Am. J. Potato Res. 89:121-128.

Posada, D., and Crandall, K. A. 2001. Evaluation of methods for detecting recombination from DNA sequences: Computer simulations. Proc. Natl. Acad. Sci. USA 98:13757-13762.

Quenouille, J., Vassilakos, N., and Moury, B. 2013. Potato virus Y: A major crop pathogen that has provided major insights into the evolution of viral pathogenicity. Mol. Plant Pathol. 14:439-452.

Rowley, J. S., Gray, S. M., and Karasev, A. V. 2015. Screening potato cultivars for new sources of resistance to Potato virus Y. Am. J. Potato Res. 92:38-48.

Salminen, M. O., Carr, J. K., Burke, D. S., and McCutchan, F. E. 1995. Identification of breakpoints in intergenotypic recombinants of HIV type 1 by Bootscanning. AIDS Res. Hum. Retroviruses 11:1423-1425.

Sanches, M. M., De Marchi, B. R., Spadotti, D. M., Nozaki, D. N., Pavan, M. A., and Krause-Sakate, R. 2014. Biological and molecular characterisation of Bidens mosaic virus supports its assignment as a member of a distinct species in the genus Potyvirus. Arch. Virol. 159:2181-2183.

Sawyer, S. A. 1999. GENECONV: A computer package for the statistical detection of gene conversion. Online publication. Department of Mathematics, Washington University, St. Louis. http://www.math.wustl.edu/ sawyer

Singh, R. P., Valkonen, J. P. T., Gray, S. M., Boonham, N., Jones, R. A. C., Kerlan, C., and Schubert, J. 2008. Discussion paper: The naming of Potato virus $Y$ strains infecting potato. Arch. Virol. 153:1-13.

Tamura, K., Stecher, G., Peterson, D., Filipski, A., and Kumar, S. 2013. MEGA6: Molecular Evolutionary Genetics Analysis version 6.0. Mol. Biol. Evol. 30:2725-2729.

Wei, T., Zhang, C., Hong, J., Xiong, R., Kasschau, K. D., Zhou, X., Carrington, J. C., and Wang, A. 2010. Formation of complexes at plasmodesmata for potyvirus intercellular movement is mediated by the viral protein P3N-PIPO. PLoS Pathog. 6:e1000962. 